\title{
Simulation and Measurement of Solar Harvesting Enhancement of Silver Plasmonic Nanoparticles on GaSb Nanodots
}

\author{
Lorenzo Rosa, ${ }^{1,2}$ Mukesh Ranjan, ${ }^{3}$ Mukul Bhatnagar, ${ }^{3}$ Daryoush Mortazavi, \\ Subroto Mukherjee, ${ }^{3}$ and Saulius Juodkazis ${ }^{1,2}$ \\ ${ }^{1}$ Applied Plasmonics (H34), Centre for Micro-Photonics, Faculty of Science, Engineering and Technology, \\ Swinburne University of Technology, Hawthorn, P.O. Box 218, Hawthorn, VIC 3122, Australia \\ ${ }^{2}$ Melbourne Centre for Nanofabrication (MCN), Australian National Fabrication Facility (ANFF), Clayton, VIC 3168, Australia \\ ${ }^{3}$ FCIPT, Institute for Plasma Research, Gandhinagar, Gujarat 382 428, India \\ ${ }^{4}$ School of Engineering, Deakin University, Geelong, VIC 3216, Australia
}

Correspondence should be addressed to Lorenzo Rosa; lrosa@ieee.org

Received 31 October 2013; Revised 12 February 2014; Accepted 26 February 2014; Published 24 March 2014

Academic Editor: Philippe Delaporte

Copyright (C) 2014 Lorenzo Rosa et al. This is an open access article distributed under the Creative Commons Attribution License, which permits unrestricted use, distribution, and reproduction in any medium, provided the original work is properly cited.

The performance of a plasmonic antireflection layer which can be utilized for deep-space radiationresistant GaSb solar cells is investigated numerically and experimentally. The layer consists of nanodots made by plasma etching of a GaSb substrate and subsequent physical vapor deposition of Ag nanoparticles on the nanodot tips, in a partially ordered configuration determined by the plasma energy level. This technique is readily applicable to patterning of silicon. We measure the substrate reflectivity and model the reflection and absorption of the substrates using the 3D finite difference time domain (FDTD) method, which are realistically imported as 3D layers from the scanning electron microscopy (SEM) images. The variation of the height of the Ag nanoparticles on top of the GaSb pillars shows that the plasmonic effect remarkably enhances the absorption. The presence of GaSb pillars enhances absorption and tunes the maximum absorption wavelength peak.

\section{Introduction}

Design and fabrication of cost-effective and efficient photovoltaic solar cells are a challenging task. Generally, nonreflective substrates like silicon are used in solar cells. These kinds of substrates exhibit high absorption and trapping of light in the solar cell substrate. However, this enhancement is confined to a short range of wavelengths. To increase the operational bandwidth, the silicon substrate is textured using etchant. This method decreases the reflectivity of the silicon substrate to the level of $20 \%$ reflection [1] and even to $1 \%$ in the case of black Si made by dry plasma etching [2].

Noble metals exhibit plasmonic effects on the surface of semiconductors and can enhance the absorption crosssection at the active region of a device by several orders of magnitude. This plasmonic effect is the result of collective oscillation of free electrons in the nanostructures when illuminated by visible-IR light. The main condition to achieve plasmonic light enhancement is deposition on noble metals patterns and layers onto a substrate. Negative electric permittivity of gold or silver in the visible-IR range is required for a particle size smaller than the incident wavelength (i.e. $a<\lambda / 10$, where $a$ is the particle size, and $\lambda$ is the incident wavelength) to create strong light field enhancement in the closest vicinity of the nanoparticle [3-5]. Noble metal nanoparticles (NPs) deposited on patterned nanostructures have shown better enhancement of the absorption crosssection in several kinds of solar cells, due to the plasmonic effect, and have given birth to the new concept of plasmonic solar cell [6-11]. Recent investigations demonstrated that partially ordered noble metal nanostructures exhibit further surface plasmon electric field enhancement up to several times [12]. By placing the NPs on pillars made from the same material as the substrate, the interaction between the metal NPs and the substrate is reduced, leading to a more pronounced Fano resonance [13]. 


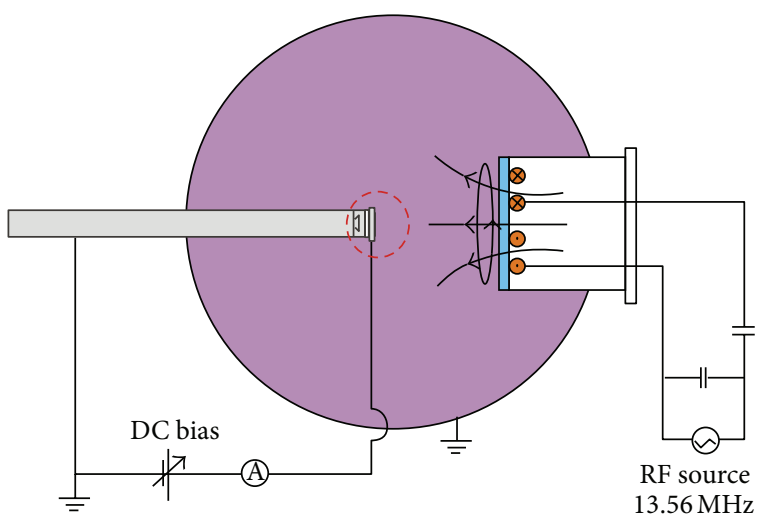

FIGURE 1: Schematic diagram of the fabrication setup: the red circle shows the sample holder immersed in radio frequency (RF) plasma; substrate sputtering is controlled via bias of a direct current (DC) source.

In this work, deep-etched GaSb substrates, forming surface nanodots (NDs), are used as the metallic NPs substrate, for application in deep-space solar cells. Following a preliminary study [14] we show, for the first time, a systematical experimental evaluation of the reflectivity enhancement given by NPs grown on NDs, which is much greater than if the particles were deposited on the bare substrate. Absorption tuning can be done through the ion energy variation which defines the size of ND. Moreover, we show by modeling further possible improvement of solar cell performance by evaluating the effect of NP height controlled by metal deposition on tips of NDs. We demonstrate an increased absorption and reduced reflection due to the plasmonic effect, which is highly desirable in solar cells.

The proposed fabrication method can be implemented on silicon substrates, but the GaSb substrates are utilized in this research because of their excellent radiation resistance capability in a wide range of visible-IR light wavelengths, which makes them promising for critically reliable deepspace operation. Metal NPs are obliquely deposited on the ND using an electron beam evaporator. This method of oblique deposition of the NPs both simplifies the fabrication process and enhances the plasmonic effect due to a partial elongation of the NPs, which makes them tuned to the polarization of the incident light [1]. The modeling of the solar cell absorption is carried out using Lumerical software which is a 3D-FDTD numerical solver of the Maxwell's equations.

\section{Fabrication}

The process to build NP-topped NDs is realized in two different phases, which control different geometrical parameters of the finished structure. In the first step, a commercial GaSb (100) substrate is epi-polished and bombarded with argon ion plasma, whose anisotropic etching produces the NDs. In the second step, a physical vapor deposition (PVD) process is used to deposit silver at grazing incidence: the ND profiles shield one another, so that the silver deposition can only happen on the top, forming separate NPs.

An ultrahigh vacuum (UHV) system was used, depicted in Figure 1, with a base vacuum level of $10^{-8}$ mbar. The coil behind the quartz window excites inductively coupled plasma, at a frequency $13.56 \mathrm{MHz}$ and a pressure of $10^{-4} \mathrm{mbar}$ of argon gas, which is accelerated on the sample holder by a potential difference. The sample is biased at a voltage varying from -600 to $-1200 \mathrm{~V}$, which permits a homogeneous plasma flow at normal incidence. Sample temperature is kept below $100^{\circ} \mathrm{C}$ to avoid temperature-induced change in the surface topography $[15,16]$. At the ion energy range used in the experiment $(600 \mathrm{eV}$ to $1200 \mathrm{eV})$, the impacting ions lead to anisotropic etching due to simultaneous surface diffusion and surface erosion of the sample [17], and a pattern of selforganized NDs is formed $[15,16,18]$, which is controlled by ion energy, fluence, and angle of incidence. The ion energy controls the height and width of the NDs.

In the second step, silver is deposited on the nanodots by e-beam evaporation, at a grazing angle of $20^{\circ}$. As the NDs partially shield one another, the deposition is maximum on the tips, where oblong-shaped NPs grow up to around $20 \mathrm{~nm}$ thickness. In this phase, it is very important to keep the flux low $(0.2 \mathrm{~nm} / \mathrm{min})$ to avoid disordered deposition [19]. Our choice of silver as the plasmonic metal is due to the fact that in this case, gold NPs show lower plasmonic effect compared to silver, due to higher interband transition loss in the visible range around $500 \mathrm{~nm}$, with lower field enhancement [20].

Figure 2 shows the end result of the partially ordered NP growth on the ND etched substrate, compared with the irregularly grown particles obtained on a polished $\mathrm{GaSb}$ sample. Atom flux is important as, while the growth happens preferentially on the tip, a high flow disrupts control on particle size and silver growth in the interdot gaps shortcircuits them. The amount of deposited silver controls NP height, while the size of the NP oblong footprint depends on the relationship between this amount and the ND width.

The experimental substrate considered for modeling is shown in Figure 3. It has been etched with ion energy of $1000 \mathrm{eV}$, obtaining NDs of around $100 \mathrm{~nm}$ size and up to $55 \mathrm{~nm}$ high, and the deposited NPs are 30 to $50 \mathrm{~nm}$ in size. The choice is due to a trade-off: increasing the ion energy increases the ion impact damage on the substrate [21] and makes the refractive index less predictable, so we chose slightly lower energy to preserve substrate quality and we show that we can make up the difference by depositing the oblique NPs, and this provides a greater insight for the numerical study.

The image FFT shown in the inset reveals a more disordered pattern than the samples previously produced (Figure 2(b)), which should help reducing reflection. This is due to the higher ion energy employed in Figure $3(1000 \mathrm{eV}$ versus $400 \mathrm{eV}$ ), because the etching process (as theoretically described by integration of the Kuramoto-Sivashinsky equation [21]) naturally delivers long-range hexagonal ordering, that is progressively disrupted by increasing the ion impact energy. The power spectral density (PSD, obtained by analysis software WSxM) of the ions in Figure 3(c) shows a noticeable 


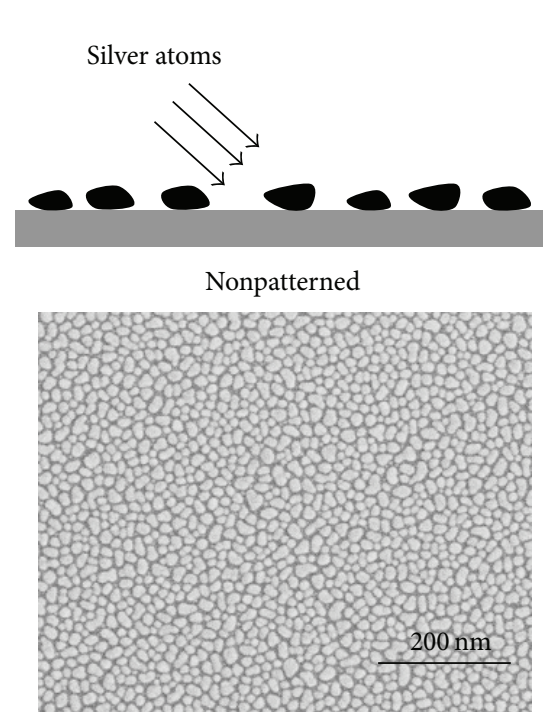

(a)

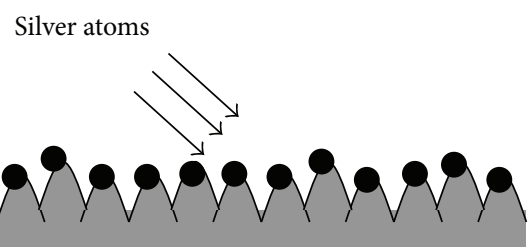

Dot patterned

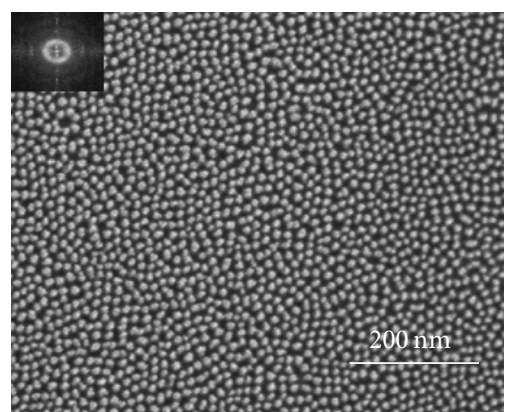

(b)

FIGURE 2: Growth of silver nanoparticles on (a) nonpatterned and (b) dot-patterned substrate. In SEM image (a), NPs are randomly distributed, while, in image (b), they show short-range hexagonal ordering (NDs are etched at ion energy of $400 \mathrm{eV}$ ). The inset shows the 2D-FFT of the image, confirming the ordering (from Rosa et al. [14]).

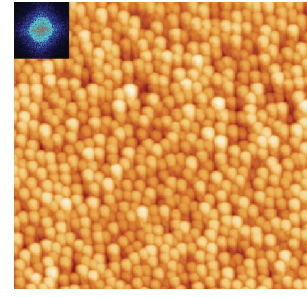

(a)

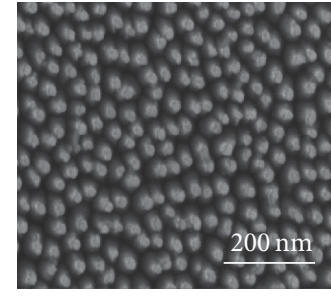

(b)

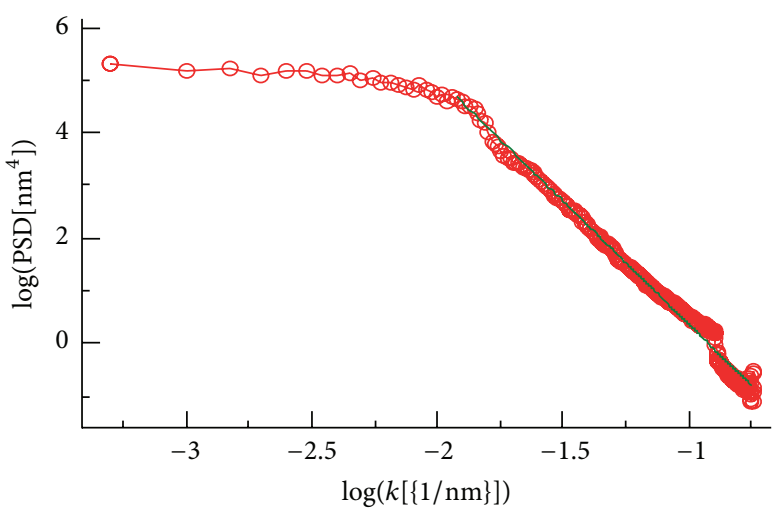

(c)

Figure 3: GaSb RIE-etched ND substrate with Ag plasmonic NPs: (a) AFM map before NP deposition (image 2D-FFT in inset shows the degree of ordering) and (b) SEM image of deposited NPs. The etching was performed at $1000 \mathrm{eV}$ ion energy, with power spectral density (PSD) shown in panel (c).

slope break around -2 (indicated by the green fitting line, and in certain cases growing into a peak [14]), which gets shifted to higher wavelengths with increasing ion energy.

\section{Experimental Results}

The results of systematical reflection measurements of samples as a function of ion energy are shown in Figure 4, with polarised light illumination at normal incidence. First a background reflection spectrum was taken from an uncoated and nonpatterned GaSb surface in the visible wavelength range of 300-700 nm and after changing the lamp, in the IR range of 700-1200 $\mathrm{nm}$ (the stitching of the two measurements is recognizable in the plot). The experimental reflection curve in Figure 4(a) for the NDs before silver deposition shows that, for increasing ion energy (and thus increasing ND size [21]), reflection decreases. Figure 4(b) shows the augmented effect after silver deposition: for ion energy of $1000 \mathrm{eV}$, the reflection curve shows two localized surface plasmon resonance (LSPR) dips corresponding to 320 and $480 \mathrm{~nm}$ wavelength, as compared with the NDs without NPs. The sample was found to be optically isotropic.

The two plasmon resonance peaks appear to be arising due to the partial ordering of the NPs, as in this case not only dipole excitation but higher ordered terms are also excited and result in two plasmon resonance peaks [22]. The $\mathrm{NP}$ footprint size and reciprocal distance is linked to the 


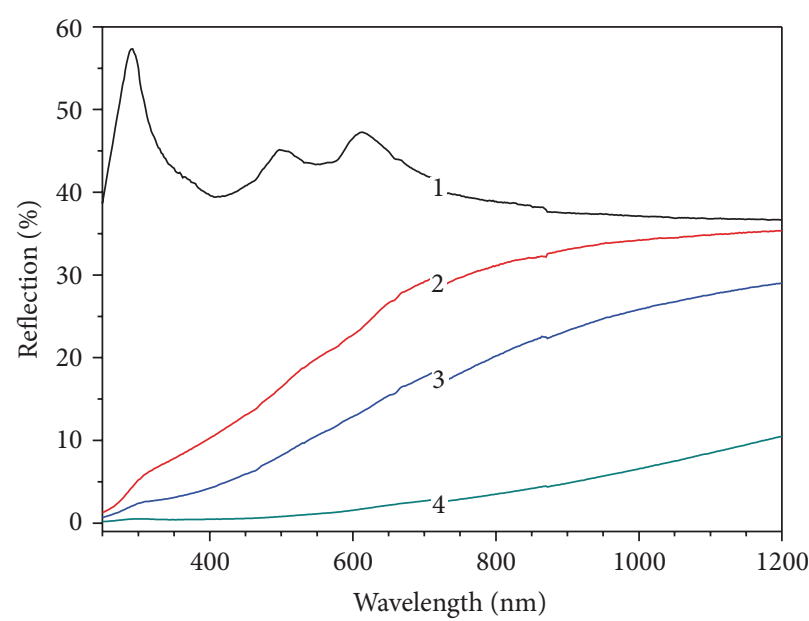

(1) Untreated

(2) $800 \mathrm{eV}$
(3) $1000 \mathrm{eV}$

(4) $1200 \mathrm{eV}$

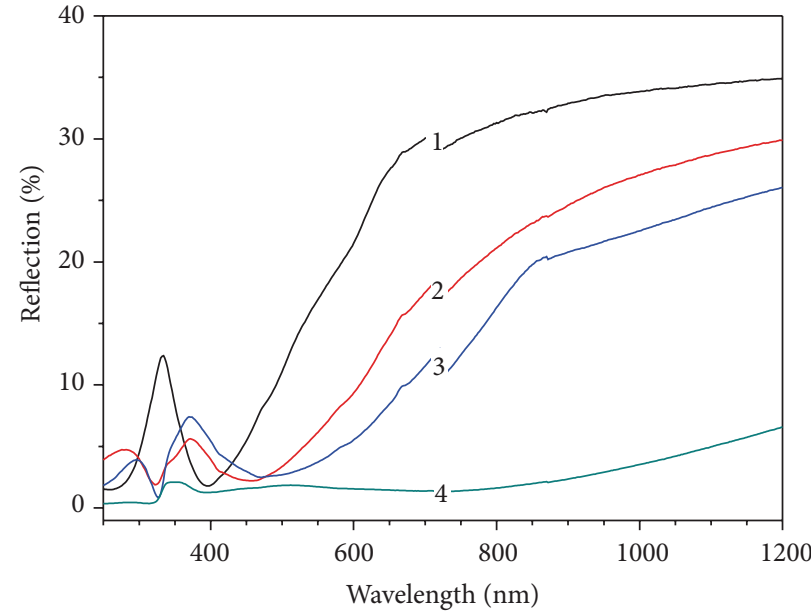

(1) $600 \mathrm{eV}$

(2) $800 \mathrm{eV}$
(3) $1000 \mathrm{eV}$

(4) $1200 \mathrm{eV}$

(a)

(b)

FIGURE 4: Experimental reflection measurements for reactive ion-etched GaSb substrates with different ion energies, (a) before and (b) after the deposition of Ag NPs on top of the NDs. The black curve in (a) refers to the untreated substrate. Spectral jumps at around $700 \mathrm{~nm}$ are artifacts due to lamp change and an automatic spectral normalization.

ND size, making longer wavelength resonance for increasing ion energy. A theoretical analysis is not possible due to the NPs nonregular and oblique shapes; however, these results are cross-checked in the 3D-FDTD simulations, when we correct the size of the particles to account for variations of experimental refractive index of $\mathrm{GaSb}$ due to ion impact [21].

\section{3D-FDTD Modelling Results and Discussion}

Nanodots are modelled by importing the SEM image of the etched GaSb substrate. Six layers of the NDs are extracted by brightness threshold in the SEM image in six levels using the limit values of $80,90,100,110,120$, and 130 , on a scale between 0 and 255. Silver NPs are also modelled from the top layer of the GaSb NDs (threshold 130) including three layers which have been shifted $4 \mathrm{~nm}$ in both $x$ - and $y$-direction to model the oblique nature of the NPs due to the slanted deposition. The average diameter of the simulated NPs varies, from around 100 to $120 \mathrm{~nm}$. The thickness of each layer of the six-layer GaSb NDs is taken as ranging from 0 to $10 \mathrm{~nm}$ (total 0 to $60 \mathrm{~nm}$ ), and the thickness of each layer of the three-layer $\mathrm{NP}$ is taken as $10 \mathrm{~nm}$.

The domain is delimited by periodic boundary conditions to avoid diffraction of the linearly polarized illuminating plane wave impinging from the top side. This makes the substrate periodic, but this has limited influence as the substrate part included is wide enough to preserve the random effect. The reflection and transmission are measured by monitors at top and bottom of the domain, at $500 \mathrm{~nm}$ distance from the etched layer, and absorption is determined by difference with the normalized source power. The inplane mesh size used is $2 \mathrm{~nm}$, which is appropriate given that the accuracy is limited by the resolution around $4 \mathrm{~nm}$ of the SEM image used in the graphical import process to generate the discretized layers. The material models are based on published experimental refractive index spectra [23, 24], which are fitted by a multicoefficient polynomial in the time domain.

The effect of ND height was explored before [14] and showed a good improvement of performance when the nonordered patterns are coupled with taller NDs in the case of cylindrical particles. Figures 5 and 6 illustrate the effect of oblong silver NPs height on the normalized absorption and reflection power, deposited on a GaAs substrate without NDs (Figure 5) and with $55 \mathrm{~nm}$ high NDs (Figure 6), which is the best performing height. The results in Figure 5 cannot be obtained experimentally and provide insight into the ND contribution, which shows that the combination of NP and ND improves reflection and absorption dramatically below $800 \mathrm{~nm}$ wavelength. A reasonable match of the features around $400 \mathrm{~nm}$ is obtained by the increased size of the NPs; however, the comparison with the experimental result in Figure 6(a) shows overestimation of the numerical reflection.

We identified four causes for the discrepancies. (1) The ion impacts disrupt the crystalline GaSb sample, so the refractive index is different from published values (NDs are covered by an amorphous layer, and preferential sputtering alters stoichiometry [21]). (2) The nanoparticle plasmonic resonance is thus shifted, and we adjusted the size for better approximation, while keeping the irregular oblong pattern constant. (3) The objective on the spectrophotometer used to measure the sample reflection has a finite numerical aperture, while the simulation result captures the scattered light on the whole reflection half-space, and thus the reflection values appear higher. (4) nanometric-sized particles show selectively 


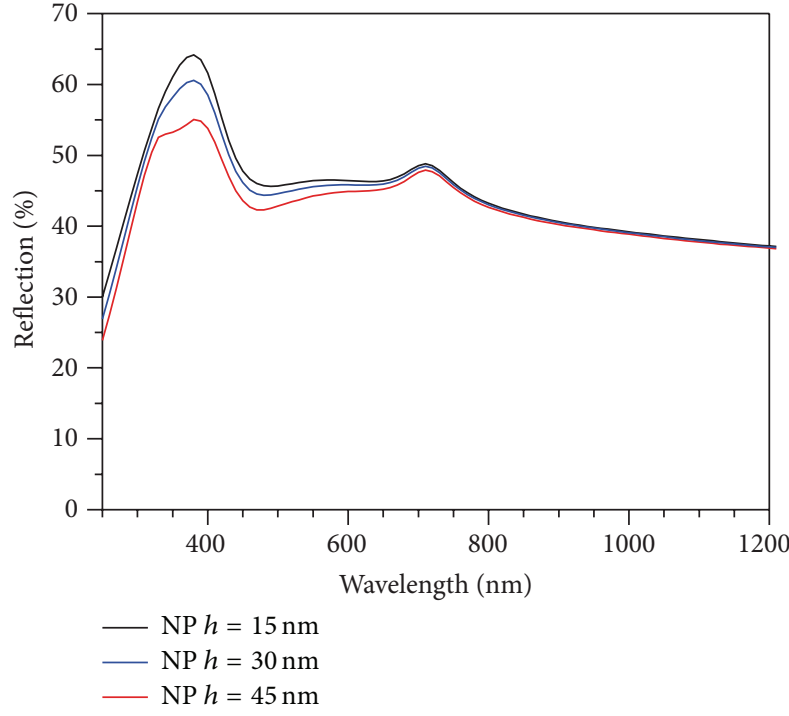

(a)

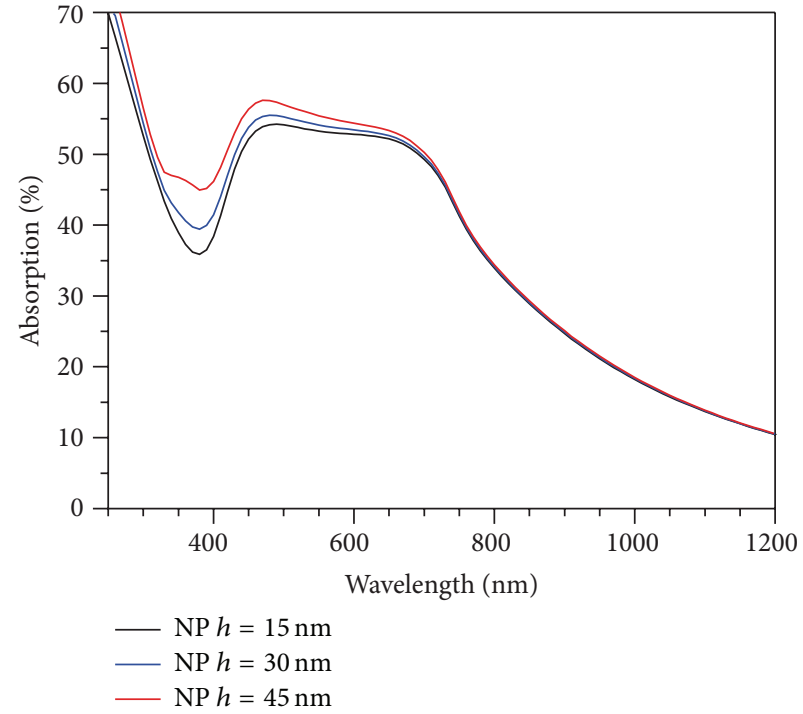

(b)

FiguRE 5: Simulated normalized (a) reflection and (b) absorption spectra of Ag NPs deposited on GaSb substrate without NDs, for varying NP height $h$.

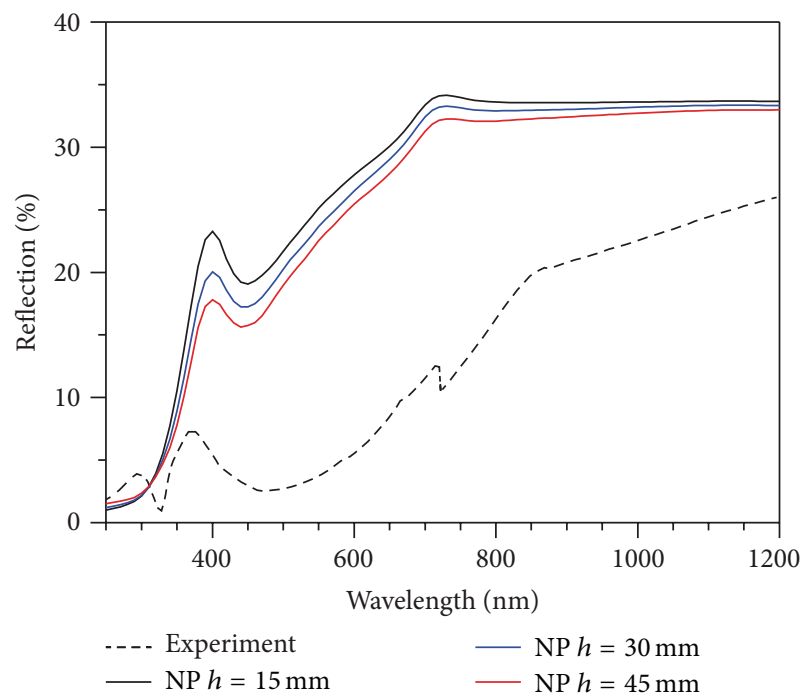

(a)

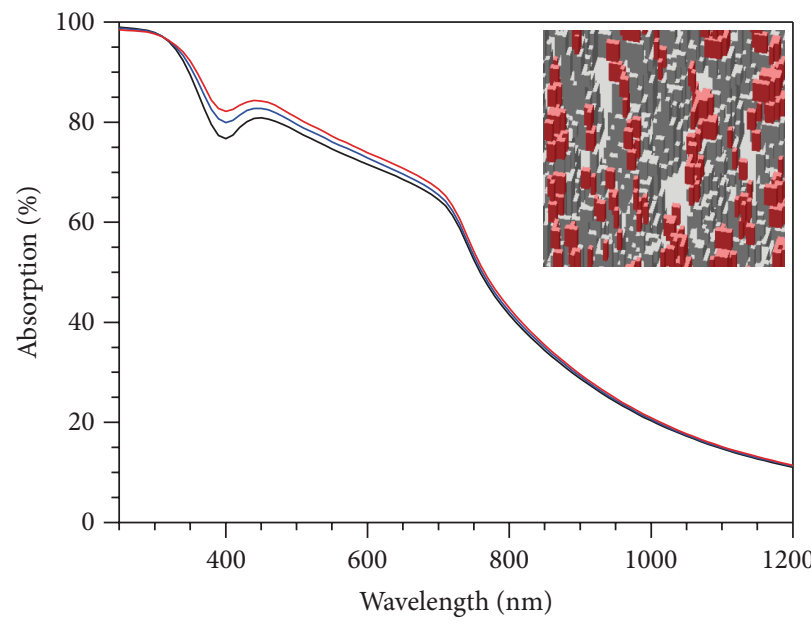

(b)

FiguRE 6: Simulated normalized (a) reflection and (b) absorption spectra of Ag NPs deposited on top of 55 nm high GaSb NDs, for varying NP height $h$. The dashed line indicates the experimental result for the $1000 \mathrm{eV}$ fabricated sample from Figure 4 . The inset shows the simulated substrate with the NPs indicated in red, emphasizing the elongation in the direction of deposition.

increased scattering for certain wavelengths depending on shape and size due to insurgence of magnetic dipole modes altering polarizability (magnetic light), as was recently shown [25] and effect is more broadband in our case as there is a distribution of shapes and sizes.

However, even in such case, it can be seen how by increasing the height of the NPs the reflection is significantly reduced, by as much as $10 \%$ for height varying from 15 to $45 \mathrm{~nm}$. The difference in absorption for the wavelengths below $600 \mathrm{~nm}$ is quite strong, in fact for the NPs deposited on the substrate without NDs (where the layer height for the
NDs is put to 0 ) as there is a $10 \%$ improvement in Figure 5(b) below $400 \mathrm{~nm}$, increasing to around $75 \%$ at $250 \mathrm{~nm}$. A similar effect is seen when the particles are deposited on top of $55 \mathrm{~nm}$ high NDs in Figure 6(b), as absorption at $400 \mathrm{~nm}$ is increased by as much as $8 \%$ by the NPs, reaching peaks of $98 \%$ around $250 \mathrm{~nm}$ wavelength. The transmission spectra at long wavelength are not significantly impacted by the size variation and are thus not shown.

We envision that further improvements can be achieved by NPs made with layered $\mathrm{Au} / \mathrm{Ag}$ alloys, in fact, as the $\mathrm{Au} / \mathrm{Ag}$ molar ratio approaches $50 \%$ and the plasmon resonance at 
near-infrared wavelength has strong variations which can be exploited to find a better performance trade-off $[26,27]$.

\section{Conclusions}

We experimentally and numerically investigated solar cell antireflective substrates based on different-height plasmonic silver NPs deposited on top of ordered dry-etched 55-nm GaSb NDs, imported in software by graphical procedure, and layered to mimic the slanted deposition process. Reflectivity reduction is given by NPs growing on NDs and is much greater than if the particles were deposited on the bare substrate. Further absorption tuning can be done through ion energy variation of ND size. The simulations show better performance for taller $45 \mathrm{~nm}$ NPs, having maximum $10 \%$ absorption improvement at wavelengths below $800 \mathrm{~nm}$, revealing a promising direction for radiation-resistant solar energy applications in space. Similar nanotextured surfaces are promising as templates for photoelectrodes and solar hydrogen applications [28-30].

\section{Conflict of Interests}

The authors declare that there is no conflict of interests regarding the publication of this paper.

\section{Acknowledgments}

Saulius Juodkazis is grateful for support via Australian Research Council DP130101205 Project Grant. Mukesh Ranjan is grateful for support via DST-Fasttrack Young Scientist Scheme (India) for the experimental work. Part of this work was performed on the swinSTAR supercomputer at Swinburne University of Technology.

\section{References}

[1] Y. Ein-Eli, N. Gordon, and D. Starosvetsky, "Reduced light reflection of textured multicrystalline silicon via NPD for solar cells applications," Solar Energy Materials and Solar Cells, vol. 90, no. 12, pp. 1764-1772, 2006.

[2] A. Žukauskas, M. Malinauskas, A. Kadys et al., "Black silicon: substrate for laser 3D micro/nano-polymerization," Optics Express, vol. 21, no. 6, pp. 6901-6909, 2013.

[3] N. Murazawa, K. Ueno, V. Mizeikis, S. Juodkazis, and H. Misawa, "Spatially selective non-linear photopolymerization induced by the near-field of surface plasmons localized on rectangular gold nanorods," Journal of Physical Chemistry C, vol. 113, no. 4-6, pp. 1147-1149, 2009.

[4] K. Ueno, S. Juodkazis, T. Shibuya, V. Mizeikis, Y. Yokota, and H. Misawa, "Nanoparticle-enhanced photopolymerization," Journal of Physical Chemistry C, vol. 113, no. 27, pp. 11720-11724, 2009.

[5] D. Mortazavi, A. Z. Kouzani, A. Kaynak, and W. Duan, "Developing LSPR design guidelines," Progress in Electromagnetics Research, vol. 126, pp. 203-235, 2012.

[6] S. Juodkazis, L. Rosa, and Y. Nishijima, "Plasmonic solutions for light harvesting in solar and sensing applications," in Nanoplasmonics: Advanced Device Applications, CRC Press, Tailor and Francis, 2013.
[7] C.-H. Poh, L. Rosa, S. Juodkazis, and P. Dastoor, "FDTD modeling to enhance the performance of an organic solar cell embedded with gold nanoparticles," Optical Materials Express, vol. 1, pp. 1326-1331, 2011.

[8] H. A. Atwater and A. Polman, "Plasmonics for improved photovoltaic devices," Nature Materials, vol. 9, no. 3, pp. 205-213, 2010.

[9] S. Vedraine, P. Torchio, A. Merlen et al., "Optical characterization of organic blend films integrating metallic nanoparticles," Solar Energy Materials and Solar Cells, vol. 102, pp. 31-35, 2011.

[10] K. Nakayama, K. Tanabe, and H. A. Atwater, "Plasmonic nanoparticle enhanced light absorption in GaAs solar cells," Applied Physics Letters, vol. 93, no. 12, Article ID 121904, 2008.

[11] S. Pillai, K. R. Catchpole, T. Trupke, and M. A. Green, "Surface plasmon enhanced silicon solar cells," Journal of Applied Physics, vol. 101, no. 9, Article ID 093105, 2007.

[12] Y. Nishijima, L. Rosa, and S. Juodkazis, "Surface plasmon resonances in periodic and random patterns of gold nano-disks for broadband light harvesting," Optics Express, vol. 20, no. 10, pp. 11466-11477, 2012.

[13] A. Dmitriev, C. Hägglund, S. Chen et al., "Enhanced nanoplasmonic optical sensors with reduced substrate effect," Nano Letters, vol. 8, no. 11, pp. 3893-3898, 2008.

[14] L. Rosa, M. Ranjan, J. Zhou et al., "Optical response simulation and measurement of silver plasmonic nano-particles in hexagonal patterns for high-efficiency solar harvesting," in Proceedings of the 50th AuSES Annual Academic Conference, PV7, Australian Solar Council, Melbourne, Australia, 2012.

[15] S. Facsko, H. Kurz, and T. Dekorsy, "Energy dependence of quantum dot formation by ion sputtering," Physical Review BCondensed Matter and Materials Physics, vol. 63, no. 16, Article ID 165329, 2001.

[16] M. A. Makeev and A.-L. Barabási, "Ion-induced effective surface diffusion in ion sputtering," Applied Physics Letters, vol. 71, no. 19, pp. 2800-2802, 1997.

[17] R. M. Bradley and J. M. E. Harper, "Theory of ripple topography induced by ion bombardment," Journal of Vacuum Science \& Technology A, vol. 6, no. 4, pp. 2390-2395, 1988.

[18] J. Zhou, S. Facsko, M. Lu, and W. Möller, "Nanopatterning of Si surfaces by normal incident ion erosion: Influence of iron incorporation on surface morphology evolution," Journal of Applied Physics, vol. 109, no. 10, Article ID 104315, 2011.

[19] M. Ranjan, T. W. H. Oates, S. Facsko, and W. Möller, "Optical properties of silver nanowire arrays with $35 \mathrm{~nm}$ periodicity," Optics Letters, vol. 35, no. 15, pp. 2576-2578, 2010.

[20] M. Ranjan and S. Facsko, "Anisotropic surface enhanced Raman scattering in nanoparticle and nanowire arrays," Nanotechnology, vol. 23, no. 48, Article ID 485307, 2012.

[21] S. Facsko, T. Bobek, T. Dekorsy, and H. Kurz, "Ordered quantum dot formation by ion sputtering," Physica Status Solidi (B), vol. 224, no. 2, pp. 537-540, 2001.

[22] A. Taleb, V. Russier, A. Courty, and M. P. Pileni, "Collective optical properties of silver nanoparticles organized in twodimensional superlattices," Physical Review B-Condensed Matter and Materials Physics, vol. 59, no. 20, pp. 13350-13358, 1999.

[23] D. E. Aspnes and A. A. Studna, "Dielectric functions and optical parameters of $\mathrm{Si}, \mathrm{Ge}, \mathrm{GaP}, \mathrm{GaAs}, \mathrm{GaSb}$, InP, InAs, and InSb from 1.5 to 6.0 eV," Physical Review B, vol. 27, no. 2, pp. 985-1009, 1983.

[24] E. D. Palik, Ed., Handbook of Optical Constants of Solids, Academic Press, San Diego, Calif, USA, 3rd edition, 1998. 
[25] A. I. Kuznetsov, A. E. Miroshnichenko, Y. H. Fu, J. B. Zhang, and B. Luk'yanchuk, "Magnetic light," Scientific Reports, vol. 2, article 492, 2012.

[26] Y. Nishijima and S. Akiyama, "Unusual optical properties of the $\mathrm{Au} / \mathrm{Ag}$ alloy at the matching mole fraction," Optical Materials Express, vol. 2, no. 9, pp. 1226-1235, 2012.

[27] E. Kowalska, Y. Nishijima, L. Rosa, and S. Juodkazis, "Silvergold nano-composites for photo-catalytic and sensing applications," in Silver Nanoparticles: Synthesis, Uses and Health Concerns, Nova Book, New York, NY, USA, 2014.

[28] K. Juodkazis, J. Juodkazytė, B. Šebeka, and S. Juodkazis, "Reversible hydrogen evolution and oxidation on Pt electrode mediated by molecular ion," Applied Surface Science, vol. 290, pp. 13-17, 2014.

[29] K. Juodkazis, J. Juodkazyte, A. Grigucevičienė, and S. Juodkazis, "Hydrogen species within the metals: role of molecular hydrogen ion $\mathrm{H}_{2}^{+}$, Applied Surface Science, vol. 258, no. 2, pp. 743-747, 2011.

[30] K. Juodkazis, J. Juodkazyte, E. Jelmakas et al., "Photoelectrolysis of water: solar hydrogen-achievements and perspectives," Optics Express, vol. 18, no. 13, pp. A147-A160, 2010. 

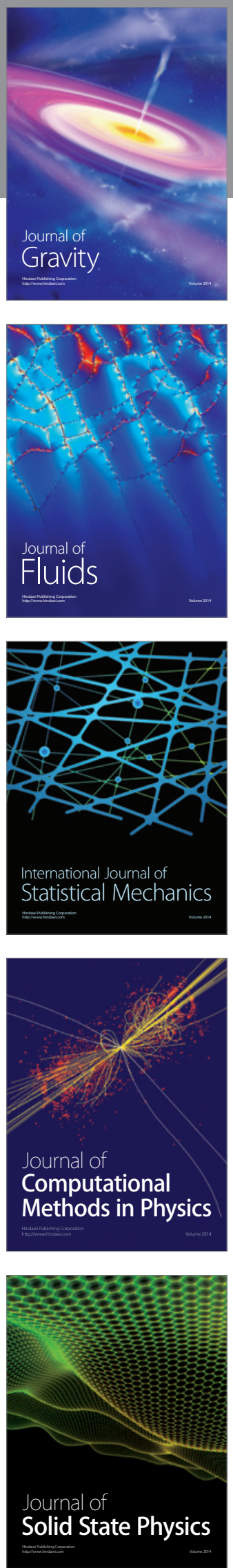

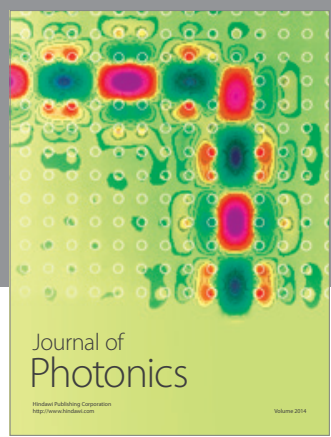

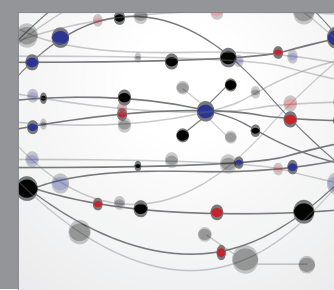

The Scientific World Journal

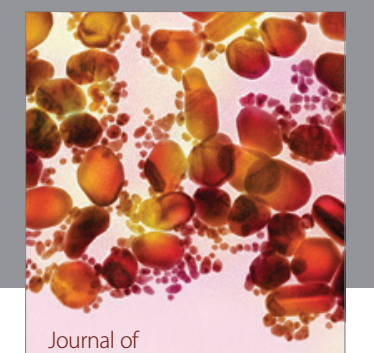

Soft Matter
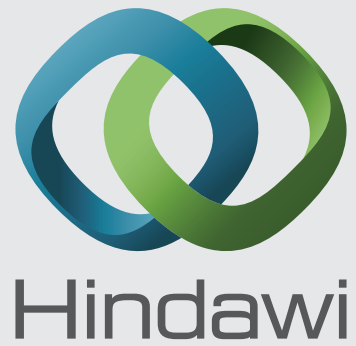

Submit your manuscripts at

http://www.hindawi.com
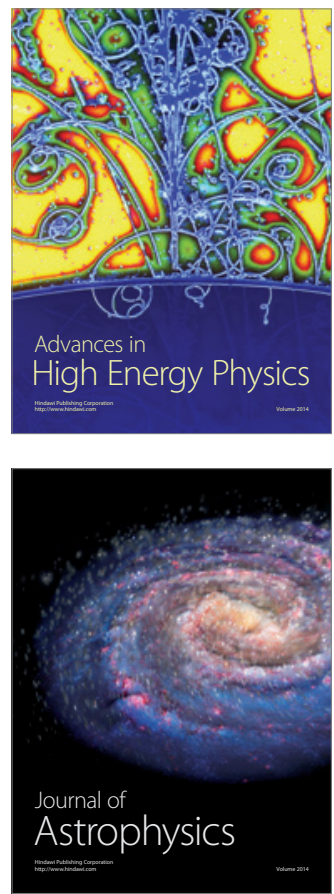
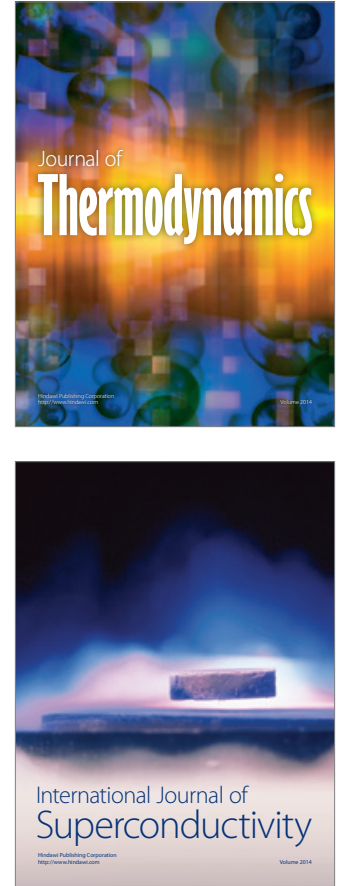
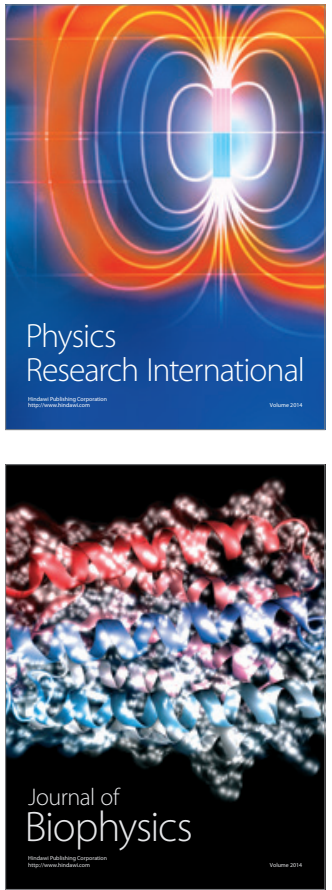
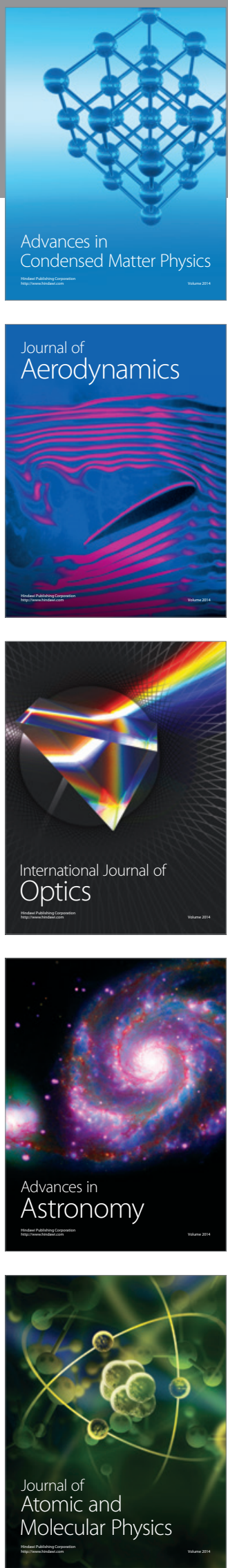